\section{Micro-optical spatial and spectral elements}

Pradeep Srinivasan, ${ }^{\text {a }}$ MEMBER SPIE, Yigit O. Yilmaz, ${ }^{\text {b }}$ MEMBER SPIE, Raymond C. Rumpf, ${ }^{c}$ MEMBER SPIE, and Eric G. Johnson ${ }^{\text {b }}$

aUniversity of Central Florida, CREOL/The College of Optics and Photonics, 4000 Central Florida Blvd, Orlando, Florida 32816

${ }^{\mathrm{b}}$ University of North Carolina at Charlotte, Center for Optoelectronics and Optical Communications, 9201 University City Blvd, Charlotte, North Carolina 28223 ${ }^{C}$ Kraetonics, LLC, 313 Hibiscus Trail, Melbourne

Beach, Florida 32951

E-mail: egjohnso@uncc.edu

Abstract. Interference filters have a defect layer incorporated within a photonic crystal structure and generate a narrow transmission notch within a wide stop band. In this paper, we propose and demonstrate wavelength-tunable spatial filters by introducing diffractive optical elements in the defect layer. The spectral transmission through the device was a function of the local defect layer thickness under broadband illumination. For each wavelength, the spatial transmission followed the contours of equal defect layer optical thickness. The devices were implemented by depositing a one-dimensional photonic crystal with a centrally integrated defect layer on a silicon substrate using plasmaenhanced chemical vapor deposition. The defect layer was lithographically patterned with charge 2, 8-level vortex structures. The spectral transmission peak and linewidth was characterized by separately illuminating each zone of diffractive element using a tunable laser source and compared with model simulations. The spatial transmission through the device was imaged onto a CCD camera. Triangular wedgeshaped zones with wavelength-dependent orientations were observed. These novel devices with spectrally tunable spatial transmission have potential applications in pupil filtering, hyperspectral imaging, and engineered illumination systems. (๑) 2009 Society of Photo-Optical Instrumentation Engineers. [DOI: $10.1117 / 1.3258651]$

Subject terms: thin-film devices; spatial filters; transmission filters; diffractive optics; integrated optics.

Paper 090605LR received Aug. 13, 2009; revised manuscript received Sep. 23, 2009; accepted for publication Sep. 25, 2009; published online Nov. 13, 2009.

\section{Introduction}

Optical interference filters have been used to achieve a transmission notch with excellent sidelobe suppression within the stop band of a photonic crystal structure. ${ }^{1}$ A one-dimensional (1-D) photonic crystal or distributed Bragg reflector consists of alternating quarter-wave-thick layers of high- and low-index materials. A transmission notch is generated when a defect is introduced into a central layer within the structure. The spectral location within the stop band is a function of the optical thickness of the defect layer. This concept has been applied to realize transmission filters for direct integration onto image sensors.

Filters with space-variant spectral transmission have been demonstrated by patterning and etching a subwave-

0091-3286/2009/\$25.00 @ 2009 SPIE length array of holes with spatially varying fill fraction through the volume of the multilayer structure. ${ }^{2,3}$ Excellent transmission tuning was achieved for a range of fill fractions. The approach has the advantage that growth and patterning/etching steps are decoupled, and it results in a simplified fabrication process. However, for small fill fractions, the effective index of the layers and their contrast reduces. The spectral linewidth of transmission becomes broader as a result. The fabrication of such filters for visible wavelengths is challenging due to the large aspect ratio of the holes that are required. ${ }^{4}$ Transmission filters for direct integration onto an image sensor have been demonstrated at visible wavelengths by spatially modulating the physical thickness of the defect layer. ${ }^{5}$ The device performs well for transmission tuning across the entire stop band since the mirrors' reflectivity remains constant. In this approach, the defect layer was patterned and etched using a $2^{N}$ patterning and etching approach that requires multiple alignment, lithography, and etching steps. This ultimately limits the number of discrete transmission wavelengths that can be obtained. Theoretically, the transmission can be tuned continuously across the stop band.

In this letter, we show that by incorporating diffractive phase functions in the defect layer, both discrete and pseudo-continuous spectral and spatial transmission tuning can be achieved. The spectral transmission notch results when the accumulated phase for the wavelength propagating through the structure equals zero (or $2 m \pi$ ). The spatial transmission profile at that spectral location corresponds to the spatial contours of equal optical thickness across the defect layer. The schematic diagram of an 8-level diffractive element with a spiral phase that was incorporated on the defect layer is shown in Fig. 1(a). The spectral transmission under broadband illumination will consist of eight discrete wavelengths corresponding to each level of the element. The spatial transmission resulting due to the element designed to have a topological charge of value $m=2$ will consist of triangular wedges separated by $180 \mathrm{deg}$ with wavelength-dependent orientation at each wavelength, as shown in Fig. 1(b) (Ref. 6). These devices represent a novel

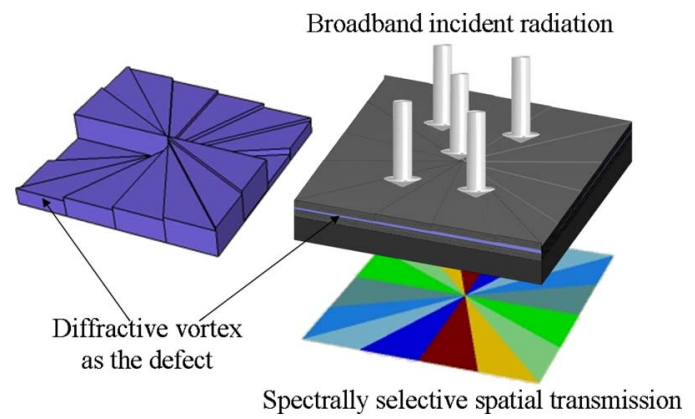

Fig. 1 (a) Schematic of a charge 2, 8-level diffractive vortex element to be incorporated in the defect layer. The optical thickness of the diffractive changes by $2 \pi$ over an angular span of $180 \mathrm{deg}$. (b) The micro-optical spatial and spectral element (MOSSE) with the vortex lens patterned and etched on the defect layer of a photonic crystal filter. The spatial transmission profile is expected to consist of triangular areas with wavelength-dependent angular orientation. (Color online only.) 


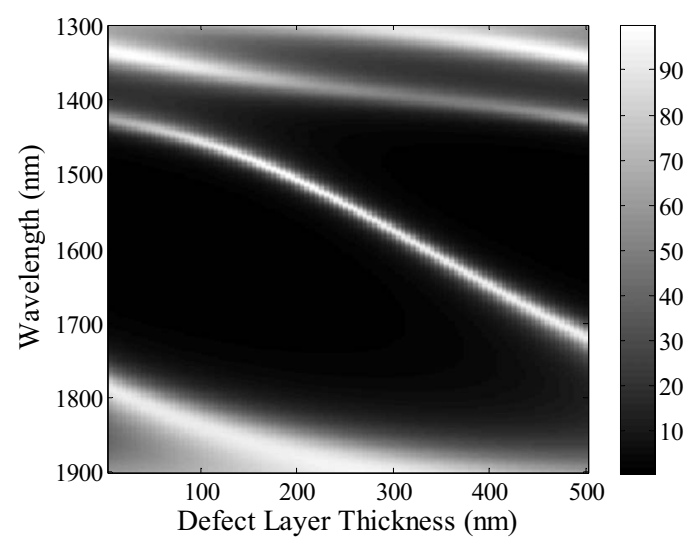

Fig. 2 Continuous tuning of the transmission as obtained across the stop band was achieved. High transmission (>90\%) and excellent sidelobe suppression was achieved throughout the tuning range. Color bar on the right indicates percent transmission.

application of interference filters, since they multiplex spatial and spectral optical functionalities and realize wavelength tunable spatial filtering.

A target structure composed of alternating layers of silicon oxide $\left(\mathrm{SiO}_{\mathrm{x}}\right)$ and nitride $\left(\mathrm{Si}_{\mathrm{x}} \mathrm{N}_{\mathrm{y}}\right)$ layers was analyzed by numerical modeling using rigorous coupled wave analysis (RCWA). ${ }^{7}$ Eight pairs of quarter-wave-thick oxide and nitride layers formed the distributed Bragg reflector (DBR) mirrors on either side of an oxide defect layer grown on a silicon substrate. In the simulation, the thickness of the defect layer was varied and the location and spectral width of the transmission notch were analyzed. Continuous transmission tuning was achieved across the photonic crystal stop band, as shown in Fig. 2. While the location of the stop band changes with defect thickness, its width was $\sim 10 \mathrm{~nm}$ throughout the tuning range. High transmission $(>90 \%)$ and excellent sidelobe suppression $(>15 \mathrm{~dB})$ were achieved across the tuning range. The linewidth can be made narrower and the suppression ratio can be improved by increasing the number of layers in the dielectric DBR or by using materials with higher index contrast.

\section{Fabrication}

In order to generate an accurate design, the refractive index of the component films was characterized across the wavelength range of interest by separately depositing the thin films of oxide and nitride using plasma-enhanced chemical vapor deposition (PECVD) on a 500- $\mu$ m-thick silicon substrate. Refractive index values of 1.4608 and 1.948 were extracted from the parameters measured on a ellipsometer at $1.55 \mu \mathrm{m}$ for the oxide and nitride films, respectively. As per design, eight pairs of 265-nm-thick silicon oxide $\left(\mathrm{SiO}_{\mathrm{x}}\right)$ and 199-nm-thick silicon nitride $\left(\mathrm{Si}_{\mathrm{x}} \mathrm{N}_{\mathrm{y}}\right)$ followed by a 450-nm-thick defect layer of $\mathrm{SiO}_{\mathrm{x}}$ were deposited using PECVD on a silicon substrate, and the wafer was removed from the growth chamber. Shipley 1813 positive photoresist was spin-coated to a thickness of $1.1 \mu \mathrm{m}$. The fabrication of diffractives on the defect layer was accomplished by the use of additive lithography on a GCA 6300 g-line stepper tool, which is a simple one-step process for fabricating multilevel optical elements. ${ }^{8}$

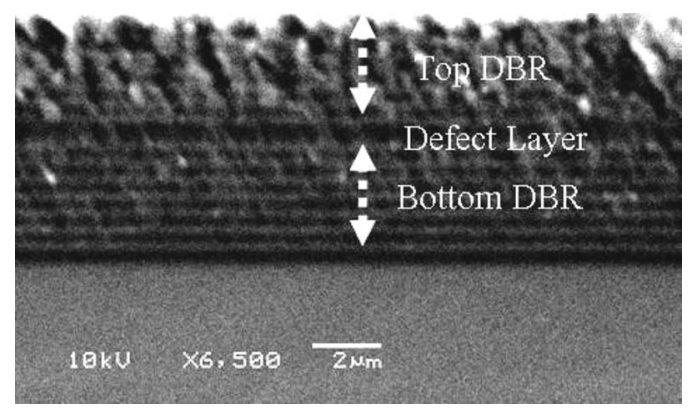

Fig. 3 Cross-sectional scanning electron micrograph (SEM) of the fabricated multilayer stack obtained by cleaving through one of the diffractive levels. The layered structure consisting of nitride (lighter regions) and oxide (darker regions) layers with an oxide defect layer is clearly seen in the micrograph.

The developed patterns were transferred into the defect layer by dry etching in $\mathrm{CHF}_{3} / \mathrm{O}_{2}$ plasma chemistry. The selectivity of etching was defined as the ratio of etched oxide to the etched photoresist. The low sag diffractives (on the order of $500 \mathrm{~nm}$ or less) were transferred using a lowselectivity process. The selectivity was controlled by changing the amount of oxygen in the plasma chemistry. Increasing amounts of oxygen in the chemistry increased the photoresist etch rate linearly but did not modify the oxide etch rate significantly. A cross-sectional scanning electron micrograph (SEM) of one of the levels of the fabricated filter is shown in Fig. 3. Heights of the levels were measured using a DekTak profilometer. The top DBR was then grown, and the device fabrication was completed.

\section{Results and Discussion}

The profilometer measured levels corresponding to each of the eight levels in the diffractive are summarized in Fig. 4(a). The heights of the diffractive levels deviated from the target heights by $10 \%$. While this change would impact the performance as a diffractive optical element, the filter performance for the intended application would not be impacted, since the transmission at these thicknesses are spaced farther than the spectral FWHM. The devices were interrogated with an unpolarized laser source that had a tuning range from $1520 \mathrm{~nm}$ to $1630 \mathrm{~nm}$ coupled to a single-mode fiber with pigtailed collimator producing a $320-\mu \mathrm{m}$ beam. Four of the vortex levels had defect layers with thicknesses appropriate for transmission in the wavelength range of the tunable laser. The results from simulation and experiment are compared in Fig. 4(b). The transmission peaks from the four diffractive levels were located at $1532 \mathrm{~nm}, 1552 \mathrm{~nm}, 1575 \mathrm{~nm}$, and $1610 \mathrm{~nm}$. The transmission line had a full width at half maximum (FWHM) of $10 \mathrm{~nm}$ in simulation and experiment. The spatial transmission through the space-variant filter element was imaged onto a CCD camera. The beam from the tunable laser was amplified using an erbium-doped fiber amplifier (EDFA), and the beam diameter was expanded to $3 \mathrm{~mm}$. This was done to ensure that large sections of the vortex element were illuminated by the beam. As expected, the transmission through the element was composed in triangular wedges separated by $180 \mathrm{deg}$ in angular space with wavelength-dependent orientation, as shown in Fig. 4(c). 


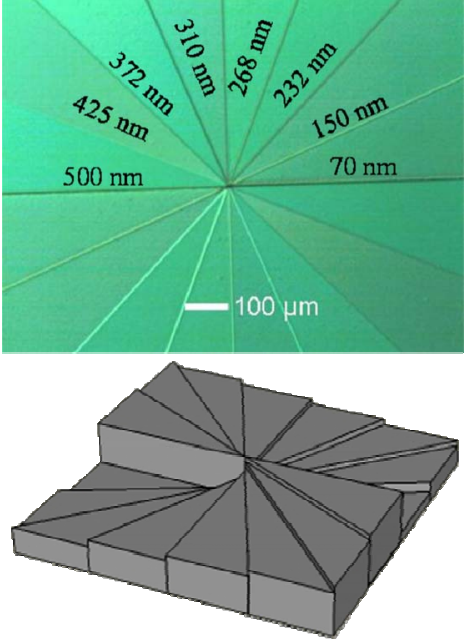

(a)

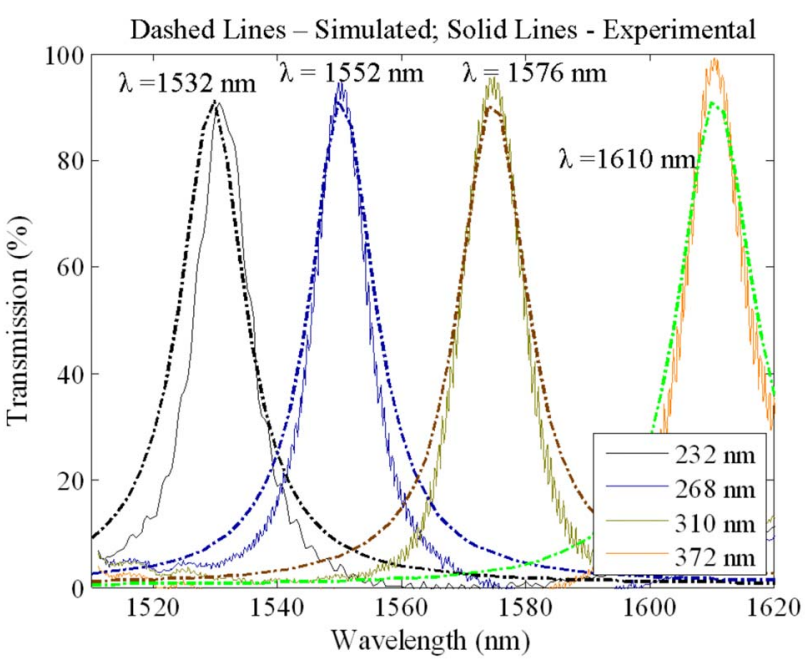

(b)

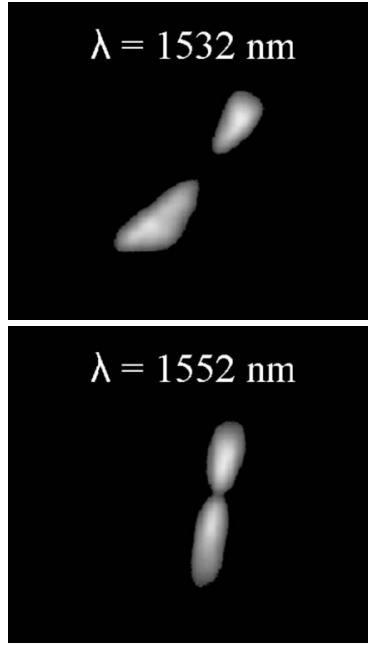

(c)

Fig. 4 (a) Optical microscope image of the charge 2 vortex elements and schematic. (b) Simulated (dotted lines) and experimental transmission spectra. (c) Images of the spatial transmission through the vortex element at $1532 \mathrm{~nm}$ and $1553 \mathrm{~nm}$. The vortex element was illuminated using a beam of diameter $3 \mathrm{~mm}$.

Wavelength-tunable spatial filters were demonstrated by integrating diffractive optical elements in the defect layer of an interference filter.

\section{Conclusions}

A novel implementation of interference filters was proposed and demonstrated in this paper. Multiplexed spatial and spectral transmission profiles were obtained by patterning diffractive optical elements in the defect layer of a photonic crystal transmission filter. The transmission characteristics were studied by simulation, and the experimental results match closely with the expected transmission profiles. Space-variant spectral transmission was achieved, and the peak location and linewidth matched the model simulations. Triangular wedges with wavelength-dependent orientation were transmitted spatially. Other transmission profiles can be obtained by incorporating appropriate phase functions on the defect layer. The devices couple spatial and spectral filtering and can be used as pupil filters for advanced multispectral imaging systems. The demonstrated devices have direct applications in other pupil filtering applications, hyperspectral imaging, and engineered illumination.

\section{Acknowledgment}

This work was funded in part through a National Science Foundation CAREER Grant (ECS0348280).

\section{References}

1. P. H. Lissberger, "Properties of all-dielectric interference filters. i. a new method of calculation," J. Opt. Soc. Am. 49(2), 121-122 (1959).

2. P. Filloux and N. Paraire, "Use of multilayer structures, periodically etched, to implement compact diffractive optical devices," J. Opt. A Pure Appl. Opt. 4(5), 175-181 (2002).

3. A. Mehta, R. C. Rumpf, Z. Roth, and E. G. Johnson, "Nanofabrication of a space-variant optical transmission filter," Opt. Lett. 31(19), 2903-2905 (2006)

4. G. Shambat, R. Athale, G. Euliss, M. Mirotznik, E. Johnson, and V. Smolski, "Reconfigurable photonic crystal filters for multi-band optical filtering on a monolithic substrate," Proc. SPIE 7041, $70410 \mathrm{P}-12$ (2008)

5. Y. Inaba, M. Kasano, K. Tanaka, and T. Yamaguchi, "Degradationfree MOS image sensor with photonic crystal color filter," IEEE Electron Device Lett. 27(6), 457-459 (2006).

6. J. H. Lee, G. Foo, E. G. Johnson, and G. A. Swartzlander Jr, "Experimental verification of an optical vortex coronagraph," Phys. Rev. Lett. 97(5), 053901 (2006).

7. M. G. Moharam, D. A. Pommet, E. B. Grann, and T. K. Gaylord, "Stable implementation of the rigorous coupled-wave analysis for surface-relief gratings: enhanced transmittance matrix approach," $J$. Opt. Soc. Am. A 12(5), 1077-1086 (1995).

8. M. Pitchumani, H. Hockel, W. Mohammed, and E. G. Johnson, "Additive lithography for fabrication of diffractive optics," Appl. Opt. 41(29), 6176-6181 (2002). 